\title{
Personality Profiles that Put Users at Risk of Perceiving Technostress
}

\author{
A Qualitative Comparative Analysis with the Big Five Personality Traits
}

\author{
Katharina Pflügner $\cdot$ Christian Maier $\cdot$ Jens Mattke $\cdot$ Tim Weitzel
}

Received: 7 June 2019/Accepted: 10 August 2020/Published online: 17 November 2020

(C) The Author(s) 2020

\begin{abstract}
Some information systems research has considered that individual personality traits influence whether users feel stressed by information and communication technologies. Personality research suggests, however, that personality traits do not act individually, but interact interdependently to constitute a personality profile that guides individual perceptions and behavior. The study relies on the differential exposure-reactivity model to investigate which personality profiles of the Big Five personality traits predispose users to perceive techno-stressors. Using a questionnaire, data was collected from 221 users working in different organizations. That data was analyzed using fuzzy set Qualitative Comparative Analysis. Based on the results, six different personality profiles that predispose to perceive high techno-stressors are identified. By investigating personality traits in terms of profiles, it is shown that a high and a low level of a personality trait can influence the perception of techno-stressors. The results will allow users and practitioners to identify individuals who are at risk of perceiving techno-stressors based on their personality profile. The post-survey analysis offers starting points for the prevention of perceived techno-
\end{abstract}

An earlier version of this paper was presented at the International Conference on Wirtschaftsinformatik, Siegen, Germany, in February 2019 (Pflügner et al. 2019).

Accepted after four revisions by Alexander Maedche.

Electronic supplementary material The online version of this article (https://doi.org/10.1007/s12599-020-00668-7) contains supplementary material, which is available to authorized users.

K. Pflügner $(\bowtie) \cdot$ C. Maier · J. Mattke $\cdot$ T. Weitzel

University of Bamberg, An der Weberei 5, 96047 Bamberg, Germany

e-mail: katharina.pfluegner@uni-bamberg.de stressors and the related negative consequences for specific personality profiles.

Keywords Technostress - Big Five personality traits . Individual differences · Prevention - Dark side of information systems - Differential exposure-reactivity model · Fuzzy set qualitative comparative analysis (fsQCA) - Configurations

\section{Introduction}

The term technostress was coined in 1984 as a modern disease (Brod 1984) and reflects stress caused by using information and communication technologies (ICTs) (Ragu-Nathan et al. 2008). Over 35 years later, technostress remains a major challenge in the modern workplace as employees are facing an increasing workload, workflow interruptions, and perceived constant availability that are associated with the ongoing digitalization (Tarafdar et al. 2019). These are examples of how ICTs stimulate technostress, which is described by so-called techno-stressors in relevant research (Ragu-Nathan et al. 2008). The "onset" of techno-stressors depends on multiple risk factors. Next to technological factors (Ayyagari et al. 2011), personality traits predispose users to perceive techno-stressors (Maier et al. 2019; Pflügner and Maier 2019). For example, neurotic individuals who tend to experience negative emotions easily, are likely to perceive technostress. Thus, the personality trait neuroticism constitutes a risk factor for succumbing to this modern disease. This explains that not all users are equally likely to become "diseased" by technostress, because personality traits influence the perception of techno-stressors. However, it fails to apply findings from other research strands suggesting that single 
personality traits lack the power to explain perceptions and that personality profiles much more strongly influence such perceptions (Vollrath and Torgersen 2000). This is supported by practical evidence which suggests the usability of personality profiles for understanding how employees perceive their work environment (Lufkin 2019). In practice, the assessment of personality profiles is widely used (Harrell 2017), because it allows managers, coworkers, and the employees themselves to recognize multiple personality traits of an employee and thereby more holistically embrace the employee.

A personality profile is an individual set of personality traits that are relevant simultaneously (Furr 2010). Personality traits act together and interdependently to influence individual perceptions (Grant and Langan-Fox 2006; Vollrath and Torgersen 2000). For example, for a personality profile of high neuroticism and low conscientiousness - a user who experiences negative emotions easily and has difficulties controlling his or her impulses - research found an association with stress. Contrary, in a personality profile of high neuroticism that is accompanied by high conscientiousness and high extraversion, the latter two personality traits can offset the negative effect of neuroticism (Vollrath and Torgersen 2000). Thus, we see that we need to study personality profiles to understand user perceptions, because some personality profiles predispose to perceptions such as stress, but others do not. Since technostress research has focused mainly on the influence of individual personality traits (Maier et al. 2019), it leaves room for comprehensively explaining the predisposing role of personality profiles and the interaction among risk factors, e.g., multiple personality traits. The contribution of one risk factor in terms of a personality trait might depend on the manifestation of another personality trait, and a high and a low level of a personality trait might influence techno-stressor perception. Based on these insights into the relevance of a user's personality traits for influencing techno-stressor perception (Maier et al. 2019) and the interaction of personality traits (Grant and Langan-Fox 2006), we aim to investigate which personality profiles predispose to perceive techno-stressors. We thus ask the research question:

Which personality profiles predispose users to perceive techno-stressors?

To answer the research question, we apply Qualitative Comparative Analysis (QCA), because this methodological approach allows us to study personality traits in profiles. Moreover, we conduct a quantitative post-survey analysis to offer initial starting points for techno-stressor prevention for specific personality profiles. Based on two arguments, we focus on the Big Five personality traits. First, the Big Five personality traits are relevant for understanding technostress, as they influence the perception of techno- stressors and the reactions to perceived techno-stressors (Maier et al. 2019; Srivastava et al. 2015). Second, the Big Five personality traits are conceptualized as core characteristics and provide a holistic picture of an individual's personality (McCrae and Costa 2008).

The paper is structured as follows: We highlight relevant research in the fields of personality and technostress first, before developing a model of the role of personality profiles in techno-stressor perception. Thereafter, we explain our methodological approach and present our empirical results. We conclude with a discussion of the results, the limitations of our study and avenues for future research.

\section{Theoretical Background}

For studying personality profiles that are relevant for techno-stressor perception, we draw on the two research streams of personality research and technostress research.

\subsection{Personality Research}

An individual's personality consists of personality traits, which are relatively enduring ways in which the individual tends to think, feel, and behave. These tendencies, i.e. personality traits, characterize an individual, distinguish individuals from one another, and predispose the individual to have various thoughts, feelings, and behaviors (Roberts et al. 2008).

Great efforts have been made to identify the specific personality traits that constitute individuals' personality. The Big Five personality traits (Costa and McCrae 1997) are typically conceptualized as core characteristics constituting the essential basis of individual differences (McCrae and Costa 2008). They thus provide a valuable reflection and holistic picture of an individual's personality. The Big Five personality traits include openness to experience, which refers to the tendency to prefer new experiences over routines. Neuroticism is the tendency to experience unpleasant emotions such as anxiety easily. Agreeableness describes the tendency to cooperate with others and conscientiousness the tendency to act in a planned and dutyoriented manner. Finally, extraversion refers to the tendency to seek the stimulation of others. These five traits are commonly seen as context-free and stable, and are useful to understand thoughts, feelings, and behaviors across different situations (Kandler et al. 2014). This means that the Big Five personality traits change little over time and influence various aspects of an individual's perceptions and behaviors. As a group, the Big Five personality traits form a personality profile, a set of coexisting Big Five personality traits. In a personality profile, the degree of each personality trait can vary widely, resulting in a virtually 
infinite number of possible personality profiles. However, only some personality profiles lead to the thoughts, feelings, or behaviors of interest in this research (Vollrath and Torgersen 2000).

The focus on a personality profile instead of individual personality traits enables the investigation of multiple coexisting personality traits to reveal their multifaceted influence and interactions. Personality literature in the fields of management or psychology inform us that it is not sufficient to focus on the effect of a single personality trait to describe and understand the effects of personality traits. Rather, the personality traits work together in leading to perceptions (Grant and Langan-Fox 2006). This means that whether a personality trait results in a perception depends on whether the other personality traits of a profile are high or low. We see these interacting effects of personality traits in related streams such as organizational research. Consider, for example, how an employee's job performance is influenced by the personality traits extraversion and conscientiousness. Research indicates that high extraversion leads to an increase in job performance among employees with a high level of conscientiousness, but to a decrease in job performance among employees with a low level of conscientiousness (Witt 2002). Thus, the simultaneous investigation of extraversion and conscientiousness reveals more information about an employee's behavior, i.e. job performance, than the separate investigation of each personality trait. Studying extraversion alone might have led to an insignificant relationship between the personality trait and job performance, which might have led to insufficient conclusions and precluded the multifaceted influence of extraversion on job performance. Similar interactive effects have been shown for further personality traits, e.g., conscientiousness and agreeableness (Witt et al. 2002), as well as other variables of interest, e.g., stressful events or counterproductive work behavior (Bardi and Ryff 2007). When investigating the influence of a personality profile on variables of interest, the interactive effects of multiple coexisting personality traits are critical. Focusing on personality profiles allows to comprehensively understand and predict an individual's perceptions and behavior based on personality traits. We find empirical support for these considerations in personality literature, where a personality profile called the Type D personality was revealed. The Type D personality is a combination of negative affectivity, which is strongly related to the Big Five personality trait neuroticism, and social inhibition, which is strongly negatively related to extraversion (Horwood et al. 2015). Research shows that this personality profile, i.e. the Type D personality, is effective in understanding and predicting how personality elicits negative consequences such as stress perception (Reich and Schatzberg 2010).

Turning to information systems (IS) literature, the Big Five personality traits were used in different contexts to understand user behavior, particularly in the context of ICT use, information technology professionals or privacy. For an overview of personality in IS literature see Maier (2012) or Online Appendix A (available online via http://link. spinger.com). Among others, it has been shown that the Big Five personality traits influence ICT acceptance, e.g., by affecting whether ICTs are perceived as useful (Devaraj et al. 2008) and whether individuals intend to use or actually use the ICT (McElroy et al. 2007). These insights are helpful to understand the relevance of the Big Five personality traits for various IS domains. However, IS literature that deals with personality has disregarded the interactive nature of coexisting personality traits. The empirical studies have investigated how separate personality traits lead to behaviors such as ICT use, but have not accounted for interactive effects or investigated personality profiles. Accounting for interactive effects in terms of personality profiles is important in order to reveal multifaceted influences of personality traits on IS relevant perceptions and behaviors. This allows to investigate under which combinations low and high levels of the Big Five personality traits lead to negative effects.

In the next section, we provide relevant background knowledge on technostress before highlighting why the investigation of personality profiles is pivotal to understand how technostress emerges.

\subsection{Technostress Research}

Technostress is a process that starts with a stimulation condition, called techno-stressor, which, when perceived, can lead to a reaction called techno-strain. Techno-stressors are stressful demands, situations or stimuli caused by ICTs that can result in techno-strain. Techno-strain refers to the consequences of perceived techno-stressors (Tarafdar et al. 2019). For a summary of relevant technostress literature, see Fischer and Riedl (2017), Tarafdar and colleagues (2019) or Online Appendix B, which includes the respective results.

Users of ICTs are confronted with a variety of technostressors, including interruptions, unreliability, and overload (Galluch et al. 2015; Gimpel et al. 2018; Riedl et al. 2012; Tams et al. 2014). Drawing from this variety, previous research has predominantly discussed five technostressors (Ragu-Nathan et al. 2008). Techno-overload describes situations in which users experience increased work volume and speed due to ICTs. Techno-invasion refers to situations where users feel the need to be permanently connected to work and where the line between 
work and personal life becomes blurred due to ICTs. Techno-complexity describes situations where ICT-related complexity leads users to feel they lack the skills and they need to spend time and effort to understand the different aspects of ICTs. Techno-insecurity refers to situations where users fear losing their job to other employees with better ICT skills or due to the replacement by an ICT. Finally, techno-uncertainty describes situations where users feel uncertainty because of ongoing changes in ICTs and where they are constantly forced to adapt, learn, and educate themselves about new ICTs (Ragu-Nathan et al. 2008).

The perception of techno-stressors can lead to technostrain, which is a non-beneficial reaction to perceived techno-stressors (Tarafdar et al. 2019). Techno-strain includes adverse behavioral reactions like low job performance or lack of innovation (Tarafdar et al. 2015). Additionally, it incorporates adverse psychological reactions such as exhaustion or job burnout (Maier et al. 2015a). Finally, perceived techno-stressors can lead to adverse physiological reactions, including increased stress hormone production (Riedl et al. 2012).

Beyond studying the reactions to perceived technostressors, some initial studies explain why an individual perceives techno-stressors and why the reactions to perceived techno-stressors arise. This ranges from technological characteristics (Ayyagari et al. 2011) to individual differences (Srivastava et al. 2015). Some technostress research has also investigated the role of a user's Big Five personality traits in perceiving technostress (see Fig. 1), drawing three primary conclusions. First, the Big Five personality traits influence users' reactions to perceived techno-stressors (Maier et al. 2015c). For example, users high on neuroticism or low on extraversion are more exhausted from using ICTs. Second, the Big Five personality traits influence how perceived techno-stressors influence job burnout and job engagement (Srivastava et al. 2015). For example, whether a user is high or low on agreeableness influences the extent to which a given level of perceived techno-stressors produces job burnout. Third, the Big Five personality trait neuroticism leads users to perceive a high level of techno-stressors (Maier et al. 2019).

In summary, IS research illustrates the detrimental effects of technostress and shows that individual personality traits influence IS-related experiences and behavior, including technostress. Building on these findings, the next section illustrates why and how personality profiles are important for comprehending why people perceive technostressors, and introduces the differential exposure-reactivity model (Bolger and Zuckerman 1995) for linking personality profiles and techno-stressors and understanding their relationship.

\section{A Model of Personality in the Technostress Process}

To understand technostress, technostress research has relied on different models and theories derived from the broader stress literature (Sonnentag and Frese 2013), like the transaction-based model of stress (Srivastava et al. 2015) and the person-environment-fit model (Ayyagari et al. 2011). We base our study on the differential exposure-reactivity model (Bolger and Zuckerman 1995), because it has been specifically developed to understand the relationship between personality and stress and provides insights into the effects of personality on technostress. The model explains how personality traits affect the perception of stressors according to the mechanism of differential exposure, and has been empirically tested in different contexts, such as stress with daily activities (Bolger and Schilling 1991), and proven stable over time (Contrada and Baum 2011). In line with the model and empirical evidence, personality affects the exposure to stressors. Exposure refers to the extent to which an individual is likely to perceive a stressor. The model posits that personality makes it either more or less likely that a person perceives stressors (Bolger and Zuckerman 1995). There is variance in exposure due to an individual's personality that is elicited because personality influences the occurrence of
Fig. 1 Research on personality traits and technostress. Note: The dotted line refers to the focus of the current study

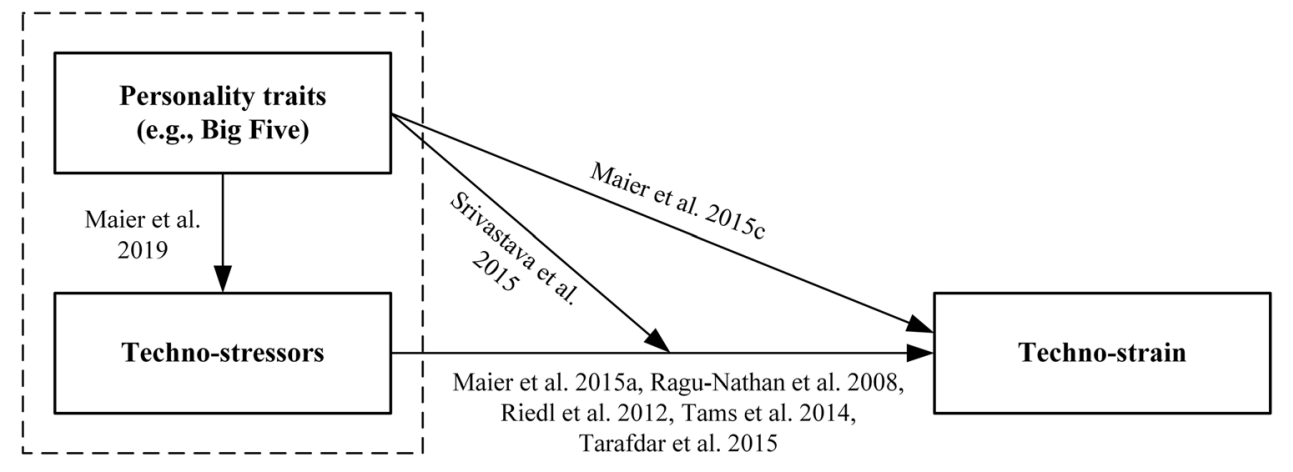


a stressor as well as the interpretation of a certain situation, stimulus or demand as stressful (Bolger and Zuckerman 1995). Regarding underlying mechanisms of differential exposure, personality traits lead individuals to certain ways of thinking and behaving that in turn create more frequent, longer, or intense stressors (Wiebe and Smith 1997). Examples of ways of thinking and behaving are characteristic styles of interaction with others, differences in executive functioning, and differences in sensitivity to threats that go in hand with personality traits like neuroticism (Contrada and Baum 2011). There is strong empirical evidence for the differential exposure process of the Big Five personality traits (Bolger and Zuckerman 1995; Contrada and Baum 2011), e.g., neuroticism increases the exposure to stressors (Grant and Langan-Fox 2007).

The current study aims to extend our understanding of the role of personality in technostress by investigating which personality profiles predispose users to perceive techno-stressors. The Big Five personality traits form distinct personality profiles, which means that every user is characterized by a set of an individual level of each of the five personality traits. We propose that personality profiles of the Big Five personality traits lead to a differential exposure to techno-stressors (see Fig. 2), because the personality profile leads users to specific ways of thinking and behaving. These include users' interactions with ICTs, their executive functioning, e.g., the control of their ICT use, or how sensitive they are to threats of an ICT, among others. We propose that personality profiles affect the extent to which a user perceives the five techno-stressors and influence the intensity of techno-stressors. We refer to that as the differential exposure effect of personality profiles on the perception of techno-stressors.

There is no prior empirical investigation into whether the differential exposure effect accounts for the perception of techno-stressors and into which personality profiles influence the technostress process, so the current study attempts to fill these gaps. The resulting propositions should help prevent users from perceiving techno-stressors and experiencing the resulting negative consequences. The following section introduces the research method we adopted to answer our research question.

\section{Method}

We next describe our methodology using fuzzy set Qualitative Comparative Analysis (fsQCA) to reveal which configurations of Big Five personality traits, i.e. personality profiles, lead to the same outcome, i.e. perceptions of techno-stressors. Each configuration is a set of the Big Five personality traits that can each be high or low.

\subsection{Data Collection}

Our sampling strategy was to survey a broad spectrum of participants who work with ICTs regularly as part of their work. We prepared an online survey and used Amazon Mechanical Turk (mTurk), which is a well-established approach in IS research, considered equal to traditional data collection approaches (Maier et al. 2019). The characteristics of the final sample are shown in Table 1.

We followed recommendations for self-reported data and tested for common method bias (CMB) and late response bias. We can conclude that $\mathrm{CMB}$ and late response bias are not a cause for concern in our data set (see Online Appendix C).

\subsection{Measures and Measurement Model}

To measure the Big Five personality traits, we used existing items, which have already been used in technostress research (Srivastava et al. 2015). Openness to experience, neuroticism, agreeableness, conscientiousness, and extraversion were measured with three items each. To measure the five techno-stressors, we used techno-overload, techno-invasion, techno-complexity, techno-insecurity, and techno-uncertainty (Ragu-Nathan et al. 2008). Techno-stressors were calculated as the sum of the five techno-stressors. All items were measured on a seven-point
Fig. 2 The influence of personality profiles on the perception of technostressors

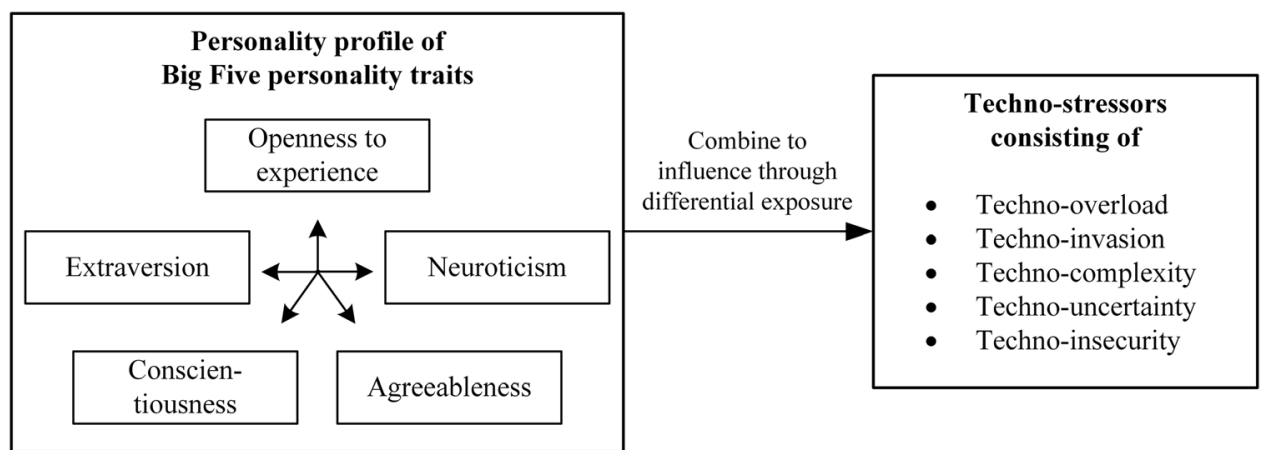


Table 1 Sample characteristics (in percent) of 221 participants

\begin{tabular}{llrlll}
\hline Age & $<20$ & 1.0 & Total ICT use & $<10$ & 10.9 \\
(in years) & $20-29$ & 40.1 & (work and private hours per week) & $10-19$ & 12.5 \\
Mean: 32.5 & $30-39$ & 40.7 & Mean: 38.6 & $20-29$ & 16.2 \\
SD: 9.1 & $40-49$ & 12.5 & SD: 19.0 & $30-39$ & 16.1 \\
& $>49$ & 5.7 & & $>39$ & 44.3 \\
Sex & Female & 39.1 & IT professional & No & 64.6 \\
& Male & 60.9 & & Yes & 35.4 \\
\hline
\end{tabular}

Likert-type agreement scale, ranging from 1 (completely disagree) to 7 (completely agree) (see Online Appendix D).

To ensure content validity, we used items that have been used and validated in previous research. We calculated the loading of each item and only used items with a loading above 0.707 (Carmines and Zeller 2008) (see Online Appendix D), thus indicator reliability is given (Carmines and Zeller 2008; Hair et al. 2019). We can assure construct reliability because the average variance extracted (AVE) of each construct is higher than 0.50 and the composite reliability (CR) is higher than 0.70 (see Online Appendix E). Furthermore, discriminant validity is given, as we calculated the heterotrait-monotrait (HTMT) ratio, which is 0.68 at most (see Online Appendix F) and consequently below the threshold of 0.85 (Hair et al. 2019). The square root of the AVE is higher than the corresponding correlations of the constructs (Fornell and Larcker 1981) (see Online Appendix E). In sum, the evaluation suggests that our data is reliable and valid.

\subsection{Data Analysis Using fsQCA}

As we aim to analyze which personality profiles predispose a user to perceive techno-stressors, we take a configurational approach. This is based on the fact that this approach allows us to study the Big Five personality traits in profiles instead of individually, and because the approach accounts for complex relationships between the distinct Big Five personality traits. We use fsQCA (Ragin 2014), which enables us to study perceived techno-stressors as the result of a sufficient configuration of personality traits. In a nutshell, fsQCA is grounded in set-theory and uses Boolean algebra to examine what configurations of conditions are sufficient configurations leading to an outcome (Ragin 2014). In this study, a sufficient configuration refers to a personality profile which predisposes to perceive a high level of techno-stressors and where each personality trait is expressed through a fuzzy set. Using fuzzy sets allows us to express the degree of membership to which a measure of a personality trait is high or low on this personality trait. This means that with fsQCA we can examine to what degree each of the five personality traits is high or low within a specific personality profile. To conduct the fsQCA analysis, we first need to calibrate the survey data into fuzzy sets.

\subsection{Calibration and Construction of the Truth Table}

As recommended in QCA literature (Mattke et al. 2020; Ortiz de Guinea and Raymond 2020), we applied the direct calibration to calibrate survey data, which was measured on a seven-point Likert scale, into fuzzy sets, which range from the value 0 to the value 1 . We followed recommendations of QCA literature (Mattke et al. 2020) and used the value 7 as the full membership anchor, which, for instance, indicates that a personality trait has a high level. We used the value 1 as the full nonmembership anchor, which, for instance, indicates that a personality trait has a low level. Finally, we used the value 4 as the crossover point. It thus indicates that a personality trait is neither high nor low (more information in Online Appendix G). We calibrated based on the Likert scale values and not based on the data distribution (e.g., taking minimum/maximum values or percentiles of the data set as anchor) for the following reasons: First, the meaning of anchors based on the data distributions is obscure and, second, bound to the specific data sample (Wagemann et al. 2016). This makes the calibration idiosyncratic to the data sample, resulting in fuzzy sets which can only be interpreted for a specific data set. This departs from the true meaning of the data from the Likert-scale which can be compared across data samples. Thus, through a calibration based on the maximum, minimum and median value of the Likert scale, we can differentiate high and low values of a personality trait, which in turn are comparable to other data sets. Based on the resulting fuzzy sets, we incorporated the calibrated data into the truth table (see Online Appendix $\mathrm{H}$ ) which lists all possible configurations of the personality traits, and calculated the consistency of each configuration. The 
consistency of a configuration (Ragin 2014) is a measure that captures the extent to which a configuration leads to the studied outcome.

\subsection{Analysis of Sufficient Configurations and Necessary Personality Traits for Perceiving a High Level of Techno-Stressors}

To reduce the truth table to sufficient configurations, we applied a frequency threshold of three (Mattke and Maier 2020), meaning that all configurations with less than three observations were dropped from further analysis. In line with QCA literature (Ragin 2009), we used a consistency threshold of 0.90 which is higher than the minimum level of 0.85 and therefore leads to better robustness of the configurations. By applying those two thresholds, we revealed sufficient configurations that predispose to perceive techno-stressors. The reduced truth table is reported in Online Appendix H. As outlined above, sufficient configuration means that individuals who show this sufficient configuration - thus a personality profile - are predisposed to perceive high techno-stressors. Finally, we applied logical minimization with the Quine-McCluskey algorithm to minimize the sufficient configurations (see Online Appendix H). The Quine-McCluskey algorithm simplifies settheoretic statements, so that logical redundant conditions can get omitted and multiple sufficient configurations can be merged into one (Ragin 2014). By applying the McCluskey algorithm we reveal more parsimonious sufficient configurations. To gain further insights about the sufficient configurations, we tested whether any of the five personality traits is necessary for perceiving high technostressors. In the context of this study, a necessary personality trait needs to exist in all configurations predisposing to perceive high techno-stressors, thus it is common that those personality traits need to exceed a consistency threshold of 0.90 and a coverage threshold of 0.60 (Mattke et al. 2020). When testing for necessity, consistency captures the extent to which a personality trait is consistently a necessary personality trait and coverage assesses the empirical relevance of the personality trait (Ragin 2006).

\section{Results}

\subsection{Main Analysis: Perception of High Techno- Stressors}

In this section, we outline the findings of the analysis of configurations sufficient for perceiving high techno-stressors. The results reveal six alternative configurations, thus six personality profiles, sufficient for predisposing to perceive high techno-stressors. We draw on the graphical illustration of the configurations for readability reasons (Fig. 3).

Configuration 1 depicts a personality profile where openness to experience plays a subordinate role and can be either high or low. Moreover, the configuration encompasses high neuroticism and agreeableness as well as low conscientiousness and extraversion. Thus, we have a user who tends to experience negative emotions easily, is more likely to want to please others, and has difficulties controlling his or her impulses, but does not seek social attention.

In Configuration 2, openness to experience can either be high or low. Moreover, it presents a configuration with high neuroticism and conscientiousness as well as low agreeableness and extraversion. Thus, we have a user who tends to experience negative emotions easily, is less likely to want to please others, is able to control his or her impulses, and does not seek social attention.

Configuration 3 encompasses a low level of openness to experience and extraversion, a don't care situation for neuroticism, as well as high levels of agreeableness and conscientiousness. Thus, we have a user who does not prefer experiences over routines nor seeks social attention, but wants to please others and is able to control his or her impulses.

Configuration 4 presents a configuration with low openness to experience as well as high neuroticism, agreeableness, and conscientiousness. Extraversion plays a subordinate role that can be either high or low. Thus, we have a user who does not prefer experiences over routines and is likely to experience negative emotions easily. Moreover, just as in Configuration 3, this user wants to please others and is able to control his or her impulses.

Configuration 5 encompasses high levels of openness to experience, agreeableness, and extraversion as well as low levels of neuroticism and conscientiousness. Thus, we have a user who prefers experiences over routines, wants to please others, and seeks social attention and other rewarding experiences. Moreover, this user does not tend to experience negative emotions easily, but has difficulties controlling his or her impulses.

Finally, Configuration 6 presents a configuration with high levels of openness to experience and neuroticism, and low levels of agreeableness and extraversion. Conscientiousness can be either high or low. Here, we have a user who prefers new experiences over routines and tends to experience negative emotions easily, but in contrast to Configuration 5 does not want to please others nor seeks social attention.

The solution coverage of 0.60 indicates the degree of how much of the outcome, i.e. high techno-stressors, is covered by the six configurations. Thus, the six configurations account for $60 \%$ of the membership in the outcome, 


\begin{tabular}{|l|c|c|c|c|c|c|c|}
\hline \multicolumn{1}{|c|}{ Configuration } & \multicolumn{2}{c|}{$\begin{array}{c}\text { Main analysis: } \\
\text { Perception of high techno-stressors }\end{array}$} & \multicolumn{2}{c|}{$\begin{array}{c}\text { Post-hoc analysis: } \\
\text { Perception of low } \\
\text { techno-stressors }\end{array}$} \\
Personality \\
trait
\end{tabular}

Note: Black circles $(\mathbf{)})$ indicate a high level of a personality trait, white crossed-out circles $(\otimes)$ a low level of a personality trait, and blank spaces indicate a 'don't care' situation. In this case, the trait plays a subordinate role and may be either high or low. C means configuration.

Fig. 3 Configurations predisposing to the perception of high techno-stressors and low techno-stressors

which illustrates a high explanatory power. The solution consistency of 0.81 is well above the minimum required consistency of 0.75 , which attests the robustness of the results. The raw coverage of the six configurations expresses the extent to which the outcome is explained by each configuration (for detailed explanation see Ragin 2006) and ranges from 0.36 to 0.38 . The comparable raw coverage scores indicate that all configurations are of equal empirical relevance for perceiving high techno-stressors. The six unique coverage values range from 0.01 to 0.03 , expressing the unique contribution of each configuration when excluding the contribution of other configurations (Ragin 2006). A comparison of the six unique coverage scores shows that each configuration has its own unique contribution to explain the perception of high techno-stressors, and the unique contribution is nearly equivalent for each configuration.

The analysis for necessary personality traits does not reveal any personality trait to be necessary. Thus, there is no single personality trait which exists in all personality profiles predisposing to perceive high techno-stressors.

In summary, we see that there are six different personality profiles which predispose to perceive high technostressors. However, this does not necessarily inform us about what personality profiles predispose to perceive low techno-stressors. QCA literature informs us about causal asymmetry (Ragin 2014), meaning that inversing the profiles predisposing to perceive high techno-stressors (e.g., inversing a high level of extraversion to a low level of extraversion) might not lead to the profiles which predispose to perceive low techno-stressors. Therefore, we next perform a post-hoc analysis and test for sufficient configurations predisposing to perceive low techno-stressors.

\subsection{Post-hoc Analysis: Perception of Low Techno- Stressors}

For this, we use the same calibrated data as above and follow the equivalent steps and thresholds. The analysis for sufficient configurations reveals two sufficient configurations of personality traits predisposing to the perception of low techno-stressors (see Fig. 3).

Configuration 7 presents a configuration with low levels of openness to experience, agreeableness, and extraversion, and with high levels of neuroticism and conscientiousness.

Configuration 8 shows a configuration with high levels of openness to experience, neuroticism, and agreeableness as well as low levels of conscientiousness and extraversion.

Comparing the results from the analysis for high and low techno-stressors, we see that Configuration 7 is the inverted version of Configuration 5, whereas Configuration 8 is not the inverted version of any of the configurations predisposing to high techno-stressors. Thus, we see that there exists causal asymmetry, meaning that inversing the profiles predisposing to perceive high techno-stressors (e.g., inversing a high level of extraversion to a low level of 
extraversion) does only partly lead to the profiles which predispose to perceive a low level of techno-stressors. The analysis for necessary personality traits does not reveal any personality trait to be necessary.

\section{Discussion}

Using ICTs can cause stress (Tarafdar et al. 2019) which starts with the perception of techno-stressors. The aim of this research is to determine which personality profiles of the Big Five personality traits predispose users to perceive techno-stressors, an insight that is relevant for understanding which users are at risk of perceiving technostressors. Taking a fsQCA approach, we identify six personality profiles that indicate a high risk of perceiving techno-stressors, and all of the six personality profiles have comparable empirical relevance for perceiving technostressors. In the following, we discuss the theoretical contributions of our research in the strand of technostress and personality, the practical implications of our findings, the limitations of our study, and possible areas for future research.

\subsection{Theoretical Contributions}

We draw on the differential exposure-reactivity model (Bolger and Zuckerman 1995) to show which personality profiles predispose a user to perceive techno-stressors. Thereby, we extend technostress literature which has so far implied that single personality traits influence users' perceptions of techno-stressors (Maier et al. 2019).

Personality profiles, consisting of the Big Five personality traits, predispose users to perceive techno-stressors. Organizational research has identified interactions between the Big Five personality traits (Witt 2002), meaning that the influence of a personality trait on an outcome depends on the level of another personality trait, e.g., because the effect of one personality trait can be buffered by another one. In line with the organizational research literature, our results show that the specific influence of a personality trait depends on the other personality traits, as we see that no personality trait is always high or always low in the configurations. Also, we reveal that all Big Five personality traits are relevant for techno-stressor perception as no personality trait plays a subordinate role in all configurations (indicated by a blank space). The post hoc analysis supports the notion that we need to take all Big Five personality traits into account because two personality traits alone do not determine whether the user perceives high or low techno-stressors and are therefore relevant for predicting health-related outcomes such as the perception of techno-stressors. We show that we can account for the interplay of all Big Five personality traits when we consider personality traits in profiles, with each personality profile consisting of a certain level of the Big Five personality traits. We contribute by revealing that investigating personality traits in profiles enables a more nuanced understanding of the interplay of personality traits predisposing to perceive high techno-stressors, which is grounded in a differential exposure (Bolger and Zuckerman 1995) to perceiving techno-stressors.

High and low levels of personality traits, depending on the level of the other personality traits of the personality profile, predispose users to perceive techno-stressors. Prior technostress literature has focused on a linear relationship between personality traits and perceived techno-stressors, i.e. a high level of neuroticism leads to a high level of perceived techno-stressors (Maier et al. 2019). Our results reveal that a high level of a personality trait can be a risk factor in one configuration but - in a different one - it can also be a low level, depending on the combination of the interdependent personality traits that constitute the personality profile. Let us consider neuroticism. Neuroticism can act in two ways, because despite the negative effect of high neuroticism (Maier et al. 2019), low neuroticism can also have a negative effect. In the case of a low level of neuroticism, when combined with low conscientiousness such as in $\mathrm{C} 5$, it makes individuals not take care of their issues that need attention (Sadahiro et al. 2015), which can provoke techno-stressor perception. We contribute that personality traits need to be interpreted relatively to the other personality traits, because drawing conclusions about a personality trait in isolation can hardly account for the finely granulated relationships.

There is not one but multiple personality profiles - including different sub-profiles of the Type D personality that predispose to techno-stressor perception. Personality literature informs us about the Type D personality that has been linked to an increased stress perception (Reich and Schatzberg 2010). In line with the findings on the Type D personality, we find the combination of high neuroticism and low extraversion in the extracted personality profiles that predispose to the perception of techno-stressors. However, we discover that there is not only one Type D personality, but different sub-profiles, e.g., one with high openness to experience (C1) and one with high agreeableness (C2). Considering also the results of the post-hoc analysis, we contribute that it is relevant to distinguish between these different Type D personality sub-profiles, because there is also a Type D sub-profile that predisposes to low techno-stressor perception. This finding suggests that Type D is not necessarily "bad", but can also lead to low techno-stressor perception.

Moreover, we extend the findings on the Type D personality by showing that there is another personality profile 
predisposing to techno-stressor perception that does not reflect the Type D personality (C5), but features a low level of conscientiousness. Recent personality literature deduces that the lack of considering conscientiousness in the Type $\mathrm{D}$ personality is a shortcoming, because conscientiousness is an important predictor for health-related outcomes (Horwood and Anglim 2017). Individuals who find it difficult to control their impulses, i.e. low level of conscientiousness, show worse health-related outcomes (Horwood and Anglim 2017). Thus, our findings support the considerations that a low level of conscientiousness is important for the prediction of health-related outcomes, i.e. the perception of techno-stressors. In addition, we extend these considerations and show that the other two Big Five personality traits, openness to experience and agreeableness, are also important for the prediction of health-related outcomes such as the perception of techno-stressors. In sum, we contribute that users with one of the identified personality profiles are prone to the modern disease of technostress, which can be a Type D sub-profile, but also a different one.

The insights into how highly perceived techno-stressors come about cannot simply be transferred to how low perceived techno-stressors come about. Considering the results of the post-hoc analysis, we can take these considerations one step further as we see that there is an asymmetric relationship between personality profiles predisposing to high and low techno-stressor perception. In line with a configurational approach, high and low levels of perceptions and behavior can be caused by diverging configurations (Ragin 2014), because high and low levels of the perception might be grounded in different mechanisms. We show that one profile predisposing to the perception of a low level of techno-stressors (C8) is different, and not just the inversion of the profiles predisposing to a high level of techno-stressors. This fact contributes to the insight that transferring the findings from a high level of techno-stressors is not sufficient to reveal how a low level of techno-stressors comes about. Instead, a separate investigation is necessary.

\subsection{Practical Implications}

This research also has implications for practice. An important step to take action to mitigate and prevent perceived techno-stressors is that employees using ICTs could assess their personality profile to evaluate their own risk level. This evaluation would enhance the employees' selfawareness concerning whether they are predisposed to perceive techno-stressors. This enhanced self-awareness is the first and an important step to prevent employees from facing techno-stressors. An assessment of the personality profile is increasingly used in organizations (Harrell 2017), e.g., for personnel development processes. Thus, information on personality profiles is becoming more and more accessible. Given that employees might share this information with their executives, our findings help executives to better understand the risk and complexity of technostressor perception. At this point, a scattergun approach is not helpful nor effective. Technostress prevention should especially target employees whose personality profile makes them especially prone to perceive techno-stressors, and the techno-stressor prevention strategies should be specific for the respective personality profile. In that way, steps for preventing techno-stressor perception would be target-oriented and focus on the risk group. Interventions that aim at mitigating that users are likely to be stressed by ICTs could target the behavioral and cognitive aspects of the personality traits (Atherton et al. 2014). Let us consider C5: This user has difficulties controlling his or her impulses, i.e. low conscientiousness. Thus, an adjustment of notifications by ICTs could decrease external impulses and thereby the difficulties. At the same time, the strengths of the personality traits of a personality profile can also be used for preventing techno-stressor perception. For instance, a conscientious user such as in $\mathrm{C} 2$ or $\mathrm{C} 3$ might very likely be able to organize and prioritize his or her ICT-related tasks which would help to reduce the perceived techno-stressors. In line with the discussion on personality profiles, we derive that applying a single measure for a personality trait, e.g., the adjustment of notifications, without considering the whole personality profile is not effective. To offer some initial starting points for technostressor prevention for specific personality profiles, we gathered data via an online questionnaire from mTurk workers. We assessed the personality profile, and the final 109 included participants match to one of the personality profiles extracted in our study. Following an explorative approach, we used open questions to ask them which technological, managerial or self-directed strategies help them to reduce their techno-stressor perception. Two researchers of the research team independently carried out the descriptive and interpretive coding (Myers 2013). We summarize the most prevalent strategies in Table 2, respecting that these initial results should be elaborated in a more comprehensive and confirmatory way in future research. In summary, we see that the exploratory approach confirms that the identified personality profiles show different strategies to prevent techno-stressor perception.

\subsection{Limitations and Further Research}

Limitations. This study is limited in some ways, but the results offer interesting room for future research. We focused on techno-stressors, which are stressful demands, situations or stimuli caused by ICTs, thus conditions 
Table 2 Strategies for the prevention of techno-stressor perception

\begin{tabular}{|c|c|}
\hline Configuration & Strategies \\
\hline Configuration 1 & $\begin{array}{l}\text { Distancing from the ICTs by switching to offline alternatives (e.g., face-to-face communication, analog formats) where } \\
\text { possible; taking a break from the ICTs (e.g., go for a walk, have a chat with colleagues) }\end{array}$ \\
\hline Configuration 2 & $\begin{array}{l}\text { Restructuring and organizing the multiple tasks (e.g., by project management technology); planning offline time to ensure } \\
\text { work-life-balance (e.g., quality time with family, airplane mode on the phone) }\end{array}$ \\
\hline Configuration 3 & $\begin{array}{l}\text { Prioritization and management of tasks (e.g., lining up with the organization's priorities, achieve short-term goals); support } \\
\text { and cooperation from/with experienced staff; improvement of ICT skills }\end{array}$ \\
\hline Configuration 4 & Provision of advanced ICTs; taking a break from the ICTs (e.g., go for a walk, social engagement with others) \\
\hline Configuration 5 & $\begin{array}{l}\text { Reduction of distractions (e.g., disable notifications, close e-mail program, shut the windows to avoid multitasking); } \\
\text { implementation of assisting ICTs (e.g., productivity monitoring software); explore the ICTs (e.g., search for advantages, } \\
\text { learn about the new ICTs); seeking assistance, counseling, and cooperation }\end{array}$ \\
\hline Configuration 6 & $\begin{array}{l}\text { Improvement of ICT skills (e.g., offering training, watching of videos); clear separation of work-related and private ICT } \\
\text { usage; realistic and gradual introduction of changes and innovation involving the employees }\end{array}$ \\
\hline
\end{tabular}

stimulating technostress (Tarafdar et al. 2019). In our research, we identify personality profiles that predispose to perceive technostress stimulating conditions. However, we did not include the adverse technostress reactions, i.e. techno-strain, in our research model. The perception of techno-stressors can lead to techno-strain, but may not always do so (Pirkkalainen et al. 2019). Thus, we cannot deduce from the findings of the current study that the extracted personality profiles also lead to techno-strain, e.g., job burnout or low job performance.

Moreover, our study is limited to profiles of the Big Five personality traits (Costa and McCrae 1997), which are the most general and stable personality traits. We chose the Big Five personality traits because they provide a holistic picture of an individual's personality (McCrae and Costa 2008) and are found to influence the perception of technostressors (Maier et al. 2019). Technostress research has also found further factors that influence the perception of techno-stressors such as demographic (e.g., country of employment) (Krishnan 2017), organizational (Tarafdar et al. 2015), task-related (Galluch et al. 2015), and technological factors (e.g., presenteeism) (Ayyagari et al. 2011), which we did not include in our research but which could affect how personality profiles predispose to technostressor perception.

We measured personality with a short scale, because the brevity allows us to reduce missing values and thereby increase the reliability, and shorter measures have also proven reliable and valid (Herzberg and Brähler 2006). Thus, our results are limited to the short scale of measuring personality, which is a proxy for multi-item scales. While we can assure the reliability and validity of our data and short scales for measuring personality have been proven an acceptable proxy of multi-item scales (Herzberg and Brähler 2006), our empirical results do not allow quality measures that compare the short scale with a multi-item scale.

Comparing our data with the data of a large representative sample of Big Five personality profiles (Gerlach et al. 2018) and a data set of Big Five personality profiles applying fsQCA as well (Maier et al. 2020) suggests that our results on personality profiles are representative and transferable. Nevertheless, there are minor differences in the frequency of personality profiles, which make it possible that there exist sparse further personality profiles predisposing to high techno-stressor perception that require a separate investigation of personality profiles in different research contexts.

Finally, we focused on perceived techno-stressors as an aggregated construct that consists of five different dimensions, i.e. techno-stressors. This approach is in line with prior technostress research (Tarafdar et al. 2015) that reached an agreement about these five techno-stressors and treated them as an overall construct. However, technostress studies have theorized and investigated specific technostressors (Ayyagari et al. 2011; Galluch et al. 2015; Maier et al. 2015b; Riedl et al. 2012). Our results are limited to the overall construct as we did not investigate the predisposition of personality profiles for each techno-stressor separately. Thus, we do not provide insights into the five specific techno-stressors as our results do not offer a precise understanding of how each specific techno-stressor emerges, although the personality profiles predisposing to a specific techno-stressor might differ.

Avenues for future research. Turning to avenues for future investigations, research could study which personality profiles predispose specifically to perceive each of the five techno-stressors and investigate the role of personality profiles for each techno-stressor separately. This might also include an examination of additional techno-stressors, as 
there are findings for additional techno-stressors, such as unreliability (Fischer et al. 2019; Riedl et al. 2012).

Moreover, our study focuses on techno-stressors that lead to negative reactions in terms of techno-strain. Recent technostress literature theorizes that techno-stressors can be divided into threat techno-stressors and challenge techno-stressors (Tarafdar et al. 2019). Threat technostressors initiate negative effects in terms of techno-strain. Challenge techno-stressors have positive effects as they lead to positive outcomes and do not initiate techno-strain. Our study focuses and is limited to the perception of threat techno-stressors. As challenge techno-stressors are different from threat techno-stressors, challenge techno-stressors might be elicited by different personality profiles than the ones extracted in our study, and future research could investigate which Big Five personality profiles lead to positive effects, i.e. predispose to the perception of challenge techno-stressors.

Finally, with fsQCA we shed light on interactions between personality traits. While fsQCA takes a configurational approach to analyze those interactions that has also proven useful in related IS research contexts (Maier et al. 2020) to study the role of personality profiles, other methods, such as decision trees or polynomial regression modeling, could be used in future research to provide further insights into the interaction and non-linear effects of personality traits.

\section{Conclusion}

Modern society is experiencing a veritable invasion of ICTs at work and in daily life, and with it techno-stressors as well as related financial costs and negative mental and physical health consequences increase. It is critical to identify at-risk users early. Taking a QCA approach, this study identifies six personality profiles of individuals predisposing to perceive techno-stressors. These findings contribute to existing research by demonstrating the relevance of considering personality profiles as indicators of a predisposition to techno-stressor perception and paving the way for future investigations of personality traits in profiles. Practitioners may use the research findings to intervene and prevent negative consequences arising from techno-stressor perception.

Open Access This article is licensed under a Creative Commons Attribution 4.0 International License, which permits use, sharing, adaptation, distribution and reproduction in any medium or format, as long as you give appropriate credit to the original author(s) and the source, provide a link to the Creative Commons licence, and indicate if changes were made. The images or other third party material in this article are included in the article's Creative Commons licence, unless indicated otherwise in a credit line to the material. If material is not included in the article's Creative Commons licence and your intended use is not permitted by statutory regulation or exceeds the permitted use, you will need to obtain permission directly from the copyright holder. To view a copy of this licence, visit http://creativecommons. org/licenses/by/4.0/.

Funding Open Access funding enabled and organized by Projekt DEAL. The project is part of the Bavarian Research Association on Healthy Use of Digital Technologies and Media (ForDigitHealth), funded by the Bavarian Ministry of Science and Arts.

\section{References}

Atherton OE, Robins RW, Rentfrow PJ, Lamb ME (2014) Personality correlates of risky health outcomes: findings from a large internet study. J Res Pers 50:56-60

Ayyagari R, Grover V, Purvis R (2011) Technostress: technological antecedents and implications. MIS Q 35(4):831-858

Bardi A, Ryff CD (2007) Interactive effects of traits on adjustment to a life transition. J Pers 75(5):955-983

Bolger N, Schilling EA (1991) Personality and the problems of everyday life: the role of neuroticism in exposure and reactivity to daily stressors. J Pers 59(3):355-386

Bolger N, Zuckerman A (1995) A framework for studying personality in the stress process. J Pers Soc Psychol 69(5):890-902

Brod C (1984) Technostress: the human cost of the computer revolution. Addison Wesley, Reading

Carmines EG, Zeller RA (2008) Reliability and validity assessment. Sage, Newbury Park

Contrada RJ, Baum A (2011) The handbook of stress science: biology, psychology, and health. Springer, New York

Costa PT, McCrae RR (1997) Set like plaster? Evidence for the stability of adult personality. In: Heatherton TF, Weinberger JL (eds) Can personality change?. American Psychological Association, Washington, pp 21-40

Devaraj S, Easley RF, Crant JM (2008) How does personality matter? Relating the five-factor model to technology acceptance and use. Inf Syst Res 19(1):93-105

Fischer T, Riedl R (2017) Technostress research: a nurturing ground for measurement pluralism? Commun Assoc Inf Syst 40(17):375-401

Fischer T, Pehböck A, Riedl R (2019) Is the technostress creators inventory still an up-to-date measurement instrument? Results of a large-scale interview study. In: Proceedings of the 14th international conference on Wirtschaftsinformatik

Fornell C, Larcker DF (1981) Evaluating structural equation models with unobservable variables and measurement error. J Market Res 18(1):39-50

Furr RM (2010) The double-entry intraclass correlation as an index of profile similarity: meaning, limitations, and alternatives. J Pers Assess 92(1):1-15

Galluch PS, Grover V, Thatcher JB (2015) Interrupting the workplace: examining stressors in an information technology context. J Assoc Inf Syst 16(1):1-47

Gerlach M, Farb B, Revelle W, Nunes Amaral LA (2018) A robust data-driven approach identifies four personality types across four large data sets. Nat Hum Behav 2(10):735-742

Gimpel H, Lanzl J, Manner-Romberg T, Nüske N (2018) Digitaler stress in Deutschland. Eine Befragung von Erwerbstätigen zu Belastung und Beanspruchung durch Arbeit mit digitalen Technologien. Working paper Forschungsförderung, Hans Böckler Stiftung (101) 
Grant S, Langan-Fox J (2006) Occupational stress, coping and strain: the combined/interactive effect of the Big Five traits. Pers Individ Differ 41(4):719-732

Grant S, Langan-Fox J (2007) Personality and the occupational stressor-strain relationship: the role of the Big Five. J Occup Health Psychol 12(1):20-33

Hair JF, Risher JJ, Sarstedt M, Ringle CM (2019) When to use and how to report the results of PLS-SEM. Eur Bus Rev 31(1):2-24

Harrell E (2017) A brief history of personality tests. https://hbr.org/ 2017/03/the-new-science-of-team-chemistry. Accessed 10 Sept 2020

Herzberg PY, Brähler E (2006) Assessing the Big-Five personality domains via short forms. Eur J Psychol Assess 22(3):139-148

Horwood S, Anglim J (2017) A critical analysis of the assumptions of Type D personality: comparing prediction of health-related variables with the Five Factor Model. Pers Individ Differ 117:172-176

Horwood S, Anglim J, Tooley G (2015) Type D personality and the Five-Factor Model: a facet-level analysis. Pers Individ Differ 83:50-54

Kandler C, Zimmermann J, McAdams DP (2014) Core and surface characteristics for the description and theory of personality differences and development. Eur J Pers 28(3):231-243

Krishnan S (2017) Personality and espoused cultural differences in technostress creators. Comput Hum Behav 66:154-167

Lufkin B (2019) The helpful upside of office personality tests. https:// www.bbc.com/worklife/article/20191030-the-helpful-upside-ofoffice-personality-tests. Accessed 10 Sept 2020

Maier C (2012) Personality within information systems research: a literature analysis. In: Proceedings of the 20th European conference on information systems (ECIS), Barcelona

Maier C, Laumer S, Eckhardt A (2015a) Information technology as daily stressor: pinning down the causes of burnout. J Bus Econ 85(4):349-387

Maier C, Laumer S, Eckhardt A, Weitzel T (2015b) Giving too much social support: social overload on social networking sites. Eur J Inf Syst 24(5):447-464

Maier C, Laumer S, Weinert C, Weitzel T (2015c) The effects of technostress and switching stress on discontinued use of social networking services: a study of Facebook use. Inf Syst J 25(3):275-308

Maier C, Laumer S, Wirth J, Weitzel T (2019) Technostress and the hierarchical levels of personality: a two-wave study with multiple data samples. Eur J Inf Syst 28(5):496-522

Maier C, Mattke J, Pflügner K, Weitzel T (2020) Smartphone use while driving: a fuzzy-set qualitative comparative analysis of personality profiles influencing frequent high-risk smartphone use while driving in Germany. Int J Inf Manag. https://doi.org/ 10.1016/j.ijinfomgt.2020.102207

Mattke J, Maier C (2020) Gamification: feature-rich mobile applications, brand awareness and loyalty. In: Proceedings of the 28th European conference on information systems (ECIS)

Mattke J, Maier C, Reis L, Weitzel T (2020) Bitcoin investment: a mixed methods study of investment motivations. Eur J Inf Syst. https://doi.org/10.1080/0960085X.2020.1787109

McCrae RR, Costa PT (2008) The five-factor theory of personality. In: John OP et al (eds) Handbook of personality: theory and research. Guilford, New York, pp 159-181

McElroy JC, Hendrickson AR, Townsend AM, DeMarie SM (2007) Dispositional factors in internet use: personality versus cognitive style. MIS Q 31(4):809-820

Myers MD (2013) Qualitative research in business and management. Sage, London
Ortiz de Guinea A, Raymond L (2020) Enabling innovation in the face of uncertainty through IT ambidexterity: a fuzzy set qualitative comparative analysis of industrial service SMEs. Int J Inf Manag 50:244-260

Pflügner K, Maier C (2019) Mitigating technostress: an empirical study of mindfulness and techno-stressors. In: Proceedings of the 25th Americas conference on information systems

Pflügner K, Mattke J, Maier C (2019) Who is stressed by using ICTs? A qualitative comparison analysis with the Big Five personality traits to understand technostress. In: Proceedings of the 14th international conference on Wirtschaftsinformatik

Pirkkalainen H, Salo M, Tarafdar M, Makkonen M (2019) Deliberate or instinctive? Proactive and reactive coping for technostress. J Manag Inf Syst 36(4):1179-1212

Ragin CC (2006) Set relations in social research: evaluating their consistency and coverage. Polit Anal 14(3):291-310

Ragin CC (2009) Qualitative comparative analysis using fuzzy sets (fsQCA). In: Rihoux B, Ragin CC (eds) Configurational comparative methods: qualitative comparative analysis (QCA) and related techniques. Sage, Los Angeles, pp 87-122

Ragin CC (2014) The comparative method: moving beyond qualitative and quantitative strategies. University of California Press, Berkeley

Ragu-Nathan TS, Tarafdar M, Ragu-Nathan BS, Tu Q (2008) The consequences of technostress for end users in organizations: conceptual development and empirical validation. Inf Syst Res 19(4):417-433

Reich J, Schatzberg A (2010) Personality traits and medical outcome of cardiac illness. J Psychiatr Res 44(15):1017-1020

Riedl R, Kindermann H, Auinger A, Javor A (2012) Technostress from a neurobiological perspective: system breakdown increases the stress hormone cortisol in computer users. Bus Inf Syst Eng 4(2):61-69

Roberts BW, Wood D, Caspi A (2008) The development of personality traits in adulthood. In: John OP et al (eds) Handbook of personality: theory and research. Guilford, New York, pp 375-398

Sadahiro R, Suzuki A, Enokido M, Matsumoto Y, Shibuya N, Kamata M, Goto K, Otani K (2015) Relationship between leukocyte telomere length and personality traits in healthy subjects. Eur Psychiatr 30(2):291-295

Sonnentag S, Frese M (2013) Stress in organizations. In: Weiner IB, Schmitt NW (eds) Handbook of psychology: industrial and organizational psychology. Wiley, Hoboken, pp 560-592

Srivastava SC, Chandra S, Shirish A (2015) Technostress creators and job outcomes: theorising the moderating influence of personality traits. Inf Syst J 25(4):355-401

Tams S, Hill K, Ortiz de Guinea A, Thatcher JB, Grover V (2014) NeuroIS - alternative or complement to existing methods? Illustrating the holistic effects of neuroscience and self-reported data in the context of technostress research. J Assoc Inf Syst 15(10):723-753

Tarafdar M, Pullins EB, Ragu-Nathan TS (2015) Technostress: negative effect on performance and possible mitigations. Inf Syst J 25(2):103-132

Tarafdar M, Cooper CL, Stich J-F (2019) The technostress trifecta techno eustress, techno distress and design: theoretical directions and an agenda for research. Inf Syst J 29(1):6-42

Vollrath M, Torgersen S (2000) Personality types and coping. Pers Individ Differ 29:367-378

Wagemann C, Buche J, Siewert MB (2016) QCA and business research: work in progress or a consolidated agenda? J Bus Res 69(7):2531-2540 
Wiebe DJ, Smith TW (1997) Personality and health: progress and problems in psychosomatics. In: Hogan R et al (eds) Handbook of personality psychology. Academic Press, San Diego, pp 891-918
Witt LA (2002) The interactive effects of extraversion and conscientiousness on performance. J Manag 28(6):835-851

Witt LA, Burke LA, Barrick MA, Mount MK (2002) The interactive effects of conscientiousness and agreeableness on job performance. J Appl Psychol 87(1):164-169 